\title{
Bessel Sequences, Riesz-Like Bases and Operators in Triplets of Hilbert Spaces
}

\author{
Giorgia Bellomonte
}

\begin{abstract}
Riesz-like bases for a triplet of Hilbert spaces are investigated, in connection with an analogous study for more general rigged Hilbert spaces performed in a previous paper. It is shown, in particular, that every $\omega$-independent, complete (total) Bessel sequence is a (strict) Riesz-like basis in a convenient triplet of Hilbert spaces. An application to non self-adjoint Schrödinger-type operators is considered. Moreover, some of the simplest operators we can define by them and their dual bases are studied.
\end{abstract}

\section{Introduction}

A Riesz basis of a Hilbert space $\mathcal{H}$ is a sequence $\left\{\xi_{n}\right\}$ of elements of $\mathcal{H}$ that are transformed into an orthonormal basis of $\mathcal{H}$ by some bounded operator with bounded inverse. Riesz bases can also be viewed as frames [1-3]; i.e., there exist positive numbers $c, C$ such that

$$
c\|\xi\|^{2} \leq \sum_{n=1}^{\infty}\left|\left\langle\xi \mid \xi_{n}\right\rangle\right|^{2} \leq C\|\xi\|^{2}, \quad \forall \xi \in \mathcal{H}
$$

What distinguishes a frame from a Riesz basis is its minimality, i.e., once one of its elements is dropped out it ceases to be a frame.

In [4] it was studied a possible extension of the notion of Riesz basis of a Hilbert space to rigged Hilbert spaces, by introducing what we called Riesz-like bases, the main difference relying on the fact that the operator transforming $\left\{\xi_{n}\right\}$ into an orthonormal basis need not to be bounded. A motivation for this generalization stems from the following considerations.

Let us assume that $\left\{\xi_{n}\right\}$ is a sequence of vectors of $\mathcal{H}$ for which there exists an unbounded closed linear operator $T$, with dense domain $D(T)$ and bounded inverse

\footnotetext{
G. Bellomonte $(\varangle)$

Dipartimento di Matematica e Informatica, Università degli Studi di Palermo,

90123 Palermo, Italy

e-mail: giorgia.bellomonte@unipa.it 
$T^{-1}$, such that $\xi_{n} \in D(T)$, for every $n \in \mathbb{N}$ and the sequence $\left\{T \xi_{n}\right\}$ is an orthonormal basis for $\mathcal{H}$. In this case we can endow $D(T) \subset \mathcal{H}$ with a new inner product

$$
\langle\xi \mid \eta\rangle_{+1}:=\langle T \xi \mid T \eta\rangle, \quad \xi, \eta \in D(T)
$$

which makes it into a Hilbert space denoted by $\mathcal{H}_{+1}$. Since $T^{-1} \in \mathcal{B}(\mathcal{H})$, the $\mathrm{C}^{*-}$ algebra of linear bounded operators in $\mathcal{H}$, it follows that $c\|\xi\| \leq\|\xi\|_{+1}$, for some $c>0$ and for every $\xi \in \mathcal{H}$, then $\mathcal{H}$ can be identified with a subspace of the conjugate dual $\mathcal{H}_{+1}^{\times}$of $\mathcal{H}_{+1}$. This space, in turn, is isomorphic to the completion of $\mathcal{H}$ with respect to the norm induced by the inner product $\langle\cdot \mid \cdot\rangle_{-1}$ defined by

$$
\langle\xi \mid \eta\rangle_{-1}:=\left\langle T^{*-1} \xi \mid T^{*-1} \eta\right\rangle, \quad \xi, \eta \in \mathcal{H} .
$$

Thus, we put $\mathcal{H}_{-1}:=\mathcal{H}_{+1}^{\times}$. Hence, the sequence $\left\{\xi_{n}\right\}$ and the operator $T$ (which is bounded from $\mathcal{H}_{+1}$ into $\mathcal{H}$, but unbounded in $\mathcal{H}$ !) automatically generate a Gelfand triplet of Hilbert spaces $\mathcal{H}_{+1} \subseteq \mathcal{H} \subseteq \mathcal{H}_{-1}$, which is a typical example of a rigged Hilbert space and we will call $\left\{\xi_{n}\right\}$ a (strict) Riesz-like basis.

A second motivation comes from the so called Pseudo-Hermitian Quantum Mechanics. This recent development of Quantum Mechanics deals with non selfadjoint Hamiltonians that often can be made into self-adjoint operators by some (generalized) similarity transformation (see, e.g., [5-8]).

Assume, in fact, that $\mathrm{H}$ is a closed operator in Hilbert space whose dense domain $D(\mathrm{H})$ is regarded as a Hilbert space $\mathcal{H}_{\mathrm{H}}$ with the graph norm $\|\cdot\|_{\mathrm{H}}$. As we will see in Sect. 2.1, this automatically produces a rigged Hilbert space. Assume that $\mathrm{H}_{s a}$ is a self-adjoint operator in $\mathcal{H}$ with discrete spectrum and, for simplicity, that each eigenvalue $\lambda_{k} \in \mathbb{R}$ has multiplicity 1 . Let $\psi_{k}$ be an eigenvector corresponding to $\lambda_{k}$. Then $\left\{\psi_{k}\right\}$ is an orthonormal basis for $\mathcal{H}$. Assume that there exists a bounded operator $T: \mathcal{H}_{H} \rightarrow \mathcal{H}$, invertible and with bounded inverse $T^{-1}: \mathcal{H} \rightarrow \mathcal{H}_{\mathrm{H}}$ such that

$$
\left\langle\mathrm{H} \xi \mid T^{\dagger} \eta\right\rangle=\left\langle T \xi \mid \mathrm{H}_{s a} \eta\right\rangle, \quad \forall \xi \in \mathcal{H}_{\mathrm{H}}, \eta \in D\left(\mathrm{H}_{s a}\right) \text { s. t. } T^{\dagger} \eta \in \mathcal{H} .
$$

Put $\xi_{k}=T^{-1} \psi_{k}$, for every $k$. Then, the set $\left\{\xi_{k}\right\}$ is a Riesz-like basis for $\mathcal{H}_{\mathrm{H}}$ and an easy computation shows that, for every $\eta \in D\left(\mathrm{H}_{s a}\right)$, such that $T^{\dagger} \eta \in \mathcal{H}$

$$
\left\langle\mathrm{H} \xi_{n} \mid T^{\dagger} \eta\right\rangle=\lambda_{n}\left\langle\xi_{n} \mid T^{\dagger} \eta\right\rangle .
$$

Thus, if $\left\{\eta \in D\left(\mathrm{H}_{s a}\right): T^{\dagger} \eta \in \mathcal{H}\right\}$ is dense in $\mathcal{H}$, we get $\mathrm{H} \xi_{n}=\lambda_{n} \xi_{n}$, for every $n$. Hence $\mathrm{H}$ has a family of eigenvectors that are mapped by $T$ into the elements of an orthonormal basis of $\mathcal{H}$. It should be noticed that the operator $T$ need not be bounded as an operator in $\mathcal{H}$. This situation is of interest because of the existence of physical models whose (non self-adjoint) Hamiltonian cannot be transformed into a self-adjoint one by similarity operators that are bounded, with bounded inverse [9].

The paper is organized as follows. In Sect. 2 we recall basic notions as that of rigged Hilbert space (RHS), of operators on a RHS, of Schauder basis and of (strict) Riesz-like basis and recall some results given in [4] about (strict) Riesz-like bases. 
In Sect. 3 we prove that every $\omega$-independent, complete (total) Bessel sequence is a strict Riesz-like basis in a convenient triplet of Hilbert spaces. Furthermore, we consider an example of application of this result to Schrödinger-type operators. In Sect. 4 we study some operators defined by strict Riesz-like bases and by their dual bases, proving their closedness, their self-adjointness and so on. We also prove that some of them are related by a weak intertwining relation and, moreover, we give a characterization of those which are quasi-Hermitian (this kind of operators are known also as pseudo-Hermitian operators in Pseudo-Hermitian Quantum Mechanics).

\section{Preliminaries and Basic Aspects}

\subsection{Rigged Hilbert Spaces and Operators on Them}

Let $\mathcal{D}$ be a dense subspace of $\mathcal{H}$. A locally convex topology $t$ on $\mathcal{D}$ finer than the topology induced by the Hilbert norm defines, in standard fashion, a rigged Hilbert space (RHS)

$$
\mathcal{D}[t] \hookrightarrow \mathcal{H} \hookrightarrow \mathcal{D}^{\times}\left[t^{\times}\right]
$$

where $\mathcal{D}^{\times}$is the vector space of all continuous conjugate linear functionals on $\mathcal{D}[t]$, i.e., the conjugate dual of $\mathcal{D}[t]$, endowed with the strong dual topology $t^{\times}=\beta\left(\mathcal{D}^{\times}, \mathcal{D}\right)$ and $\hookrightarrow$ denotes a continuous embedding. Since the Hilbert space $\mathcal{H}$ can be identified with a subspace of $\mathcal{D}^{\times}\left[t^{\times}\right]$, we will systematically read (3) as a chain of topological inclusions: $\mathcal{D}[t] \subset \mathcal{H} \subset \mathcal{D}^{\times}\left[t^{\times}\right]$. In this paper we will consider only the case where $\mathcal{D}$ itself is a Hilbert space, denoted by $\mathcal{H}_{+1}$, under a norm stronger than that of $\mathcal{H}$. Its conjugate dual is denoted by $\mathcal{H}_{-1}$.

As an example, let us be given a closed operator $T$ with dense domain $D(T)$ in Hilbert space $\mathcal{H}$. Then, a rigged Hilbert space, more precisely a triplet of Hilbert spaces, arises in a natural way. Indeed, the domain $D(T)$ with the graph norm $\|\cdot\|_{T}$ defined by

$$
\|\xi\|_{T}=\left(\|\xi\|^{2}+\|T \xi\|^{2}\right)^{1 / 2}=\left\|(I+T * T)^{1 / 2} \xi\right\|, \quad \xi \in D(T)
$$

becomes a Hilbert space, namely $\mathcal{H}_{T}$. If $\mathcal{H}_{T}^{\times}$denotes the Hilbert space conjugate dual of $\mathcal{H}_{T}$, then we get the triplet of Hilbert spaces

$$
\mathcal{H}_{T} \subset \mathcal{H} \subset \mathcal{H}_{T}^{\times}
$$

If $\mathcal{H}$ and $\mathcal{K}$ are two Hilbert spaces, we will indicate by $\mathcal{B}(\mathcal{H}, \mathcal{K})$ the Banach space of linear bounded operators in $\mathcal{H}$ into $\mathcal{K}$. If $\mathcal{H}=\mathcal{K}$, then, to simplify the notation, we will put $\mathcal{B}(\mathcal{H}, \mathcal{H})=\mathcal{B}(\mathcal{H})$.

Let $\mathcal{H}_{+1} \subset \mathcal{H} \subset \mathcal{H}_{-1}$ be a triplet of Hilbert spaces. An involution $X \mapsto X^{\dagger}$ can be introduced in $\mathcal{B}\left(\mathcal{H}_{+1}, \mathcal{H}_{-1}\right)$ by the equality 


$$
\left\langle X^{\dagger} \eta \mid \xi\right\rangle=\overline{\langle X \xi \mid \eta\rangle}, \quad \forall \xi, \eta \in \mathcal{H}_{+1} .
$$

Hence $\mathcal{B}\left(\mathcal{H}_{+1}, \mathcal{H}_{-1}\right)$ is a ${ }^{\dagger}$-invariant vector space.

\subsection{Schauder, Riesz-Like and Strict Riesz-Like Bases}

Let $\mathcal{E}\left[t_{\mathcal{E}}\right]$ be a locally convex vector space and $\left\{\xi_{n}\right\}$ a sequence of vectors of $\mathcal{E}$. We adopt the following terminology:

(i) the sequence $\left\{\xi_{n}\right\}$ is complete or total if the linear span of $\left\{\xi_{n}\right\}$ is dense in $\mathcal{E}\left[t_{\mathcal{E}}\right]$;

(ii) the sequence $\left\{\xi_{n}\right\}$ is $\omega$-independent if $\sum_{n=1}^{\infty} c_{n} \xi_{n}=0$, implies $c_{n}=0$, for every $n \in \mathbb{N}$;

(iii) the sequence $\left\{\xi_{n}\right\}$ is a topological basis for $\mathcal{E}$ if, for every $\phi \in \mathcal{E}$, there exists a unique sequence $\left\{c_{n}\right\}$ of complex numbers such that

$$
\phi=\sum_{n=1}^{\infty} c_{n} \xi_{n},
$$

where the series on the right hand side converges in $\mathcal{E}\left[t_{\mathcal{E}}\right]$.

(iv) a topological basis $\left\{\xi_{n}\right\}$ for $\mathcal{E}\left[t_{\mathcal{E}}\right]$ is a Schauder basis if the coefficient functionals $\left\{c_{n}=c_{n}(f)\right\}$, appearing in (4), are $t_{\mathcal{E}}$-continuous.

If $\left\{\xi_{n}\right\}$ is a topological basis for $\mathcal{E}$, then $\left\{\xi_{n}\right\}$ is $\omega$-independent and therefore it consists of linearly independent vectors. Moreover, in Banach spaces, the two notions of topological basis and of Schauder basis do coincide.

In the remainder of the paper the Hilbert space $\mathcal{H}$ will always be meant as a separable one.

Consider a rigged Hilbert space $\mathcal{D}[t] \subset \mathcal{H} \subset \mathcal{D}^{\times}\left[t^{\times}\right]$and a Schauder basis $\left\{\xi_{n}\right\}$ for $\mathcal{D}[t]$. Every $f \in \mathcal{D}$ is the sum of a series $\sum_{n=1}^{\infty} c_{n}(f) \xi_{n}$, with uniquely determined, suitable coefficients $c_{n}(f)$. By the continuity of the linear functionals $c_{n}$ on $\mathcal{D}[t]$, it follows the existence and the uniqueness of a sequence $\left\{\zeta_{n}\right\} \subset \mathcal{D}^{\times}$such that

$$
c_{n}(f)=\overline{\left\langle\zeta_{n} \mid f\right\rangle}, \quad \forall n \in \mathbb{N}, f \in \mathcal{D} \text {. }
$$

If we take $f=\xi_{k}$, then $c_{n}\left(\xi_{k}\right)=\overline{\left\langle\zeta_{n} \mid \xi_{k}\right\rangle}=\delta_{n, k}$ i.e., the two sequences $\left\{\xi_{n}\right\}$ and $\left\{\zeta_{n}\right\}$ are biorthogonal.

The following statements on Schauder bases, given here only for triplet of Hilbert spaces $\mathcal{H}_{+1} \subset \mathcal{H} \subset \mathcal{H}_{-1}$, was proved in [4] for general rigged Hilbert spaces by adapting results given in $[2,10,11]$.

Proposition 2.1 Let $\left\{\xi_{n}\right\}$ be a Schauder basis for $\mathcal{H}_{+1}$. Then there exists a unique sequence $\left\{\zeta_{n}\right\}$ of vectors of $\mathcal{H}_{-1}$ such that 
(i) the sequences $\left\{\xi_{n}\right\}$ and $\left\{\zeta_{n}\right\}$ are biorthogonal;

(ii) for every $f \in \mathcal{H}_{+1}$,

$$
f=\sum_{n=1}^{\infty} \overline{\left\langle\zeta_{n} \mid f\right\rangle} \xi_{n}
$$

(iii) The partial sum operator $S_{n}$, given by

$$
S_{n} f=\sum_{k=1}^{n} \overline{\left\langle\zeta_{k} \mid f\right\rangle} \xi_{k}, \quad f \in \mathcal{H}_{+1}
$$

is continuous from $\mathcal{H}_{+1}$ into $\mathcal{H}_{+1}$ and has an adjoint $S_{n}^{\dagger}$ everywhere defined in $\mathcal{H}_{-1}$ given by

$$
S_{n}^{\dagger} \Psi=\sum_{k=1}^{n}\left\langle\Psi \mid \xi_{k}\right\rangle \zeta_{k}, \quad \Psi \in \mathcal{H}_{-1} ;
$$

(iv) the sequence $\left\{\zeta_{n}\right\}$ is a basis for $\mathcal{H}_{-1}$ with respect to the weak topology; i.e., if $\Psi \in \mathcal{H}_{-1}$ one has

$$
\langle\Psi \mid f\rangle=\left\langle\sum_{k=1}^{\infty}\left\langle\Psi \mid \xi_{k}\right\rangle \zeta_{k} \mid f\right\rangle=\sum_{k=1}^{\infty}\left\langle\Psi \mid \xi_{k}\right\rangle\left\langle\zeta_{k} \mid f\right\rangle, \quad \forall f \in \mathcal{H}_{+1}
$$

Remark 2.2 Of course, (6) provides a weak expansion for every $h \in \mathcal{H}$; i.e., $h=$ $\sum_{k=1}^{\infty}\left\langle h \mid \xi_{k}\right\rangle \zeta_{k}$, weakly. In particular, for $f \in \mathcal{H}_{+1} \subset \mathcal{H}_{-1}$, (6) gives

$$
\|f\|^{2}=\sum_{k=1}^{\infty}\left\langle f \mid \xi_{k}\right\rangle\left\langle\zeta_{k} \mid f\right\rangle, \quad \forall f \in \mathcal{H}_{+1}
$$

so that the series on the right hand side is convergent, for every $f \in \mathcal{H}_{+1}$.

Now we recall the notion of Riesz-like and strict Riesz-like bases we gave in [4] for a rigged Hilbert space $\mathcal{D}[t] \subset \mathcal{H} \subset \mathcal{D}^{\times}\left[t^{\times}\right]$.

Definition 2.3 A Schauder basis $\left\{\xi_{n}\right\}$ for $\mathcal{D}[t]$ is called a Riesz-like basis for $\mathcal{D}[t]$ if there exists a one-to-one continuous operator $T: \mathcal{D}[t] \rightarrow \mathcal{H}$ such that $\left\{T \xi_{n}\right\}$ is an orthonormal basis for $\mathcal{H}$.

The range $R(T)$ of $T$ contains the orthonormal basis $\left\{e_{k}\right\}$ with $e_{k}:=T \xi_{k}, k \in \mathbb{N}$, hence $R(T)$ is dense in $\mathcal{H}$.

If $\left\{\xi_{n}\right\}$ is a Riesz-like basis, we can find explicitly the sequence $\left\{\zeta_{n}\right\} \subset \mathcal{H}_{-1}$ of Proposition 2.1. The continuity of $T$ and (5), in fact, imply

$$
T f=\sum_{n=1}^{\infty} \overline{\left\langle\zeta_{n} \mid f\right\rangle} T \xi_{n}=\sum_{n=1}^{\infty} \overline{\left\langle\zeta_{n} \mid f\right\rangle} e_{n}, \quad \forall f \in \mathcal{H}_{+1}
$$


This, in turn, implies that $\overline{\left\langle\zeta_{n} \mid f\right\rangle}=\left\langle T f \mid e_{n}\right\rangle$, for every $f \in \mathcal{H}_{+1}$. Hence $\zeta_{n}=T^{\dagger} e_{n}$, for every $n \in \mathbb{N}$.

Clearly, for every $n, k \in \mathbb{N}$,

$$
\left\langle\zeta_{k} \mid \xi_{n}\right\rangle=\left\langle T^{\dagger} e_{k} \mid \xi_{n}\right\rangle=\left\langle e_{k} \mid T \xi_{n}\right\rangle=\left\langle e_{k} \mid e_{n}\right\rangle=\delta_{k, n}
$$

and $T^{\dagger} T \xi_{n}=\zeta_{n}$, for every $n \in \mathbb{N}$. This sequence is called the $d u a l$ sequence.

Let $\left\{\xi_{n}\right\}$ be a Riesz-like basis for $\mathcal{D}[t]$. One can ask what happens if we strengthen the hypotheses on $T$, e.g. if we suppose that $T$ is onto too and $T^{-1}$ is continuous from $\mathcal{H}$ into $\mathcal{D}[t]$. In other words, let us suppose that the operator $T$ which makes of $\left\{T \xi_{n}\right\}$ an orthonormal basis for $\mathcal{H}$ has a continuous inverse $T^{-1}: \mathcal{H}[\|\cdot\|] \rightarrow \mathcal{D}[t]$ (in particular, $T^{-1}$ is a bounded operator in $\mathcal{H}$ ). We say in this case that $\left\{\xi_{n}\right\}$ is a strict Riesz-like basis for $\mathcal{D}[t]$. This assumption has important consequences on the involved topologies. Indeed, as shown in [4, Proposition 3.6]

Proposition 2.4 If the rigged Hilbert space $\mathcal{D}[t] \subset \mathcal{H} \subset \mathcal{D}^{\times}\left[t^{\times}\right]$, with $\mathcal{D}[t]$ complete and reflexive, has a strict Riesz-like basis $\left\{\xi_{n}\right\}$ then it is (equivalent to) a triplet of Hilbert spaces $\mathcal{H}_{+1} \subset \mathcal{H} \subset \mathcal{H}_{-1}$. Moreover, $\left\{\xi_{n}\right\}$ is an orthonormal basis for $\mathcal{H}_{+1}$ and the dual sequence $\left\{\zeta_{n}\right\}$ is an orthonormal basis for $\mathcal{H}_{-1}$.

In other words the rigged Hilbert space is forced to be a triplet of Hilbert spaces. On the other hand, in a triplet of Hilbert spaces $\mathcal{H}_{+1} \subset \mathcal{H} \subset \mathcal{H}_{-1}$, if the operator $T$ which makes of $\left\{T \xi_{n}\right\}$ an orthonormal basis for $\mathcal{H}$ is onto, then $T^{-1}$ is automatically continuous and so the basis $\left\{\xi_{n}\right\}$ is strict.

Remark 2.5 It is clear that, if $\left\{\xi_{n}\right\}$ is a strict Riesz-like basis, then it is an unconditional basis of $\mathcal{H}_{+1}$.

\section{Bessel Sequences as Strict-Riesz Like Bases}

Now, we will give an answer to the following natural questions: given a sequence $\left\{\xi_{n}\right\} \subset \mathcal{H}$, does there exist a rigged Hilbert space such that $\left\{\xi_{n}\right\}$ is a strict Riesz-like basis for it? Given a sequence $\left\{\xi_{n}\right\} \subset \mathcal{H}$, does there exist a triplet of Hilbert spaces $\mathcal{H}_{+1} \subseteq \mathcal{H} \subseteq \mathcal{H}_{-1}$ such that $\left\{\xi_{n}\right\}$ is an orthonormal basis for $\mathcal{H}_{+1}$ ?

Let $\left\{\xi_{n}\right\}$ be a Bessel sequence in $\mathcal{H}$, i.e., [11] there exists $C>0$ such that for every finite sequence of complex numbers $\left\{c_{1}, c_{2}, \ldots c_{n}\right\}, n \in \mathbb{N}$,

$$
\left\|\sum_{k=1}^{n} c_{k} \xi_{k}\right\|^{2} \leq C \sum_{k=1}^{n}\left|c_{k}\right|^{2} .
$$

Let $\left\{e_{n}\right\}$ be an orthonormal basis for $\mathcal{H}$ and define the operator

$$
V: \sum_{k=1}^{n} c_{k} e_{k} \rightarrow \sum_{k=1}^{n} c_{k} \xi_{k} .
$$


It is clear that $V$ is well-defined and bounded (by (7)) on $\mathcal{G}=\operatorname{span}\left\{e_{n}\right\}$, then it extends to a bounded operator, denoted again by $V$, to $\mathcal{H}$. Obviously, $V e_{n}=\xi_{n}$, for every $n \in \mathbb{N}$.

We notice that $\left\{\xi_{n}\right\}$ is $\omega$-independent if and only if $V$, and $\left\{\xi_{n}\right\}$ is complete if and only if $V^{*}$ is injective. Now we give our main result.

Theorem 3.1 If $\left\{\xi_{n}\right\}$ is an $\omega$-independent complete Bessel sequence in $\mathcal{H}$, then, for every orthonormal basis $\left\{e_{n}\right\}$ of $\mathcal{H}$, there exists a triplet of Hilbert spaces $\mathcal{K} \subset \mathcal{H} \subset$ $\mathcal{K}^{\times}$which has $\left\{\xi_{n}\right\}$ as a strict Riesz-like basis. This triplet is unique up to unitary transformations.

Proof We maintain the notations of the previous discussion. Let $\left\{e_{n}\right\}$ be an orthonormal basis of $\mathcal{H}$. If $\left\{\xi_{n}\right\}$ is a $\omega$-independent Bessel sequence in $\mathcal{H}$, the operator $V$ defined in (8) is injective on $\mathcal{H}$. Indeed, since $\left\{e_{n}\right\}$ is an orthonormal basis for $\mathcal{H}$, for every $f \in \mathcal{H}, f=\lim _{N \rightarrow \infty} f_{N}$, where $f_{N}=\sum_{n=1}^{N}\left\langle f \mid e_{n}\right\rangle e_{n} \in \mathcal{G}$. It follows that

$$
V f:=\lim _{N \rightarrow \infty} V f_{N}=\lim _{N \rightarrow \infty} \sum_{n=1}^{N}\left\langle f \mid e_{n}\right\rangle \xi_{n}=\sum_{n=1}^{\infty}\left\langle f \mid e_{n}\right\rangle \xi_{n}
$$

If $V f=0$, then $\left\langle f \mid e_{n}\right\rangle=0$, for every $n \in \mathbb{N}$. Hence $f=0$.

Then $V$ has an inverse $V^{-1}$ defined on the range $\operatorname{Ran}(V)$ of $V$ and, since $V$ is bounded, $V^{-1}$ is closed. Moreover, $\left\{\xi_{n}\right\} \subseteq \operatorname{Ran}(V)$, hence, by the completeness of $\left\{\xi_{n}\right\}$, the inverse of the operator $V$ is densely defined. Now, we have

$$
\operatorname{Ran}(V)=\left\{g \in \mathcal{H}: g=\sum_{n=1}^{\infty} c_{n} \xi_{n} \text { with } \sum_{n=1}^{\infty}\left|c_{n}\right|^{2}<\infty\right\}
$$

The $\omega$-independence of $\left\{\xi_{n}\right\}$ guarantees the uniqueness of the expansion $g=$ $\sum_{n=1}^{\infty} c_{n} \xi_{n}$ of every $g \in \operatorname{Ran}(V)$. Finally, we have

$$
V^{-1} g=V^{-1}\left(\sum_{n=1}^{\infty} c_{n} \xi_{n}\right)=\sum_{n=1}^{\infty} c_{n} e_{n}, \quad \forall g=\sum_{n=1}^{\infty} c_{n} \xi_{n} \in \operatorname{Ran}(V)
$$

We put, for short, $T:=V^{-1}$ and $D(T)=\operatorname{Ran}(V)$. Then $T$ is a closed densely defined operator such that $T \xi_{n}=e_{n}$ and has bounded inverse. Then, as we have already seen in Sect.2.1, a triplet of Hilbert spaces arises in a natural way. More precisely, we get the triplet of Hilbert spaces

$$
\mathcal{H}_{T} \subset \mathcal{H} \subset \mathcal{H}_{T}^{\times}
$$

where $\mathcal{H}_{T}=D(T)\left[\|\cdot\|_{T}\right]$ with

$$
\|\xi\|_{T}=\left(\|\xi\|^{2}+\|T \xi\|^{2}\right)^{1 / 2}=\left\|\left(I+T^{*} T\right)^{1 / 2} \xi\right\|, \quad \xi \in D(T)
$$


and the sequence $\left\{\xi_{n}\right\}$ is a strict Riesz-like basis for $\mathcal{H}_{T}$. Now, let us consider two different orthonormal bases $\left\{e_{n}\right\}$ and $\left\{e_{n}^{\prime}\right\}$ of the Hilbert space $\mathcal{H}$. Then, as it is wellknown, there exists a unitary operator $U: \mathcal{H} \rightarrow \mathcal{H}$ such that $U e_{n}=e_{n}^{\prime}$, therefore the two norms $\|\cdot\|_{T}$ and $\|\cdot\|_{U T}$ coincide and hence the two Hilbert spaces $\mathcal{H}_{T}$ and $\mathcal{H}_{U T}$ do.

Remark 3.2 If $\left\{\xi_{n}\right\}$ is an $\omega$-independent complete Bessel sequence in $\mathcal{H}$, then, for every orthonormal basis $\left\{e_{n}\right\}$ of $\mathcal{H}$, there exists a (unique) Hilbert space which has $\left\{\xi_{n}\right\}$ as an orthonormal basis since, once the triplet of Hilbert spaces $\mathcal{H}_{T} \subset \mathcal{H} \subset \mathcal{H}_{T}^{\times}$ is at hand (Theorem 3.1), then $\left\{\xi_{n}\right\}$ is an orthonormal basis for $\mathcal{H}_{+1}=D(T)\left[\|\cdot\|_{+1}\right]$ and, as a consequence of the uniqueness of $\mathcal{H}_{T}$, the Hilbert space $\mathcal{H}_{+1}$ (and the triplet), is unique too.

Remark 3.3 If $T=V^{-1}$ is also bounded, then $\left\{\xi_{n}\right\}$ is a Riesz basis for $\mathcal{H}$ and $\mathcal{H}_{T}$ coincides with $\mathcal{H}$ as a vector space but it carries a different albeit equivalent norm, as stated by the well-known theory of Riesz bases.

Remark 3.4 If $\left\{\xi_{n}\right\}$ is an $\omega$-independent complete Bessel sequence in $\mathcal{H}$, then Theorem 3.1 gives us full information on the possibility of expanding a vector $f \in \mathcal{H}$ in terms of $\left\{\xi_{n}\right\}$ : indeed, $\left\{\xi_{n}\right\}$ determines a closed densely defined operator $T$ and every vector $f$ of the domain of $T$ can be expanded uniquely as an unconditionally convergent series $f=\sum_{n=1}^{\infty} c_{n} \xi_{n}$, the convergence holds in the graph norm $\|\cdot\|_{T}$ of $D(T)$, and then in the norm $\|\cdot\|$. Other vectors $f$ of $\mathcal{H}$, by (6), can be obtained by a weakly convergent series $f=\sum_{k=1}^{\infty}\left\langle f \mid \xi_{k}\right\rangle \zeta_{k},\left\{\zeta_{k}\right\}$ being the dual sequence of $\left\{\xi_{n}\right\}$, in the sense that $\langle f \mid \eta\rangle=\sum_{k=1}^{\infty}\left\langle f \mid \xi_{k}\right\rangle\left\langle\zeta_{k} \mid \eta\right\rangle, \forall \eta \in D(T)$.

If $T$ is unbounded, then $0 \in \sigma_{c}\left(T^{-1}\right)$, the continuous spectrum of $T^{-1}$. Some more information on $\left\{\xi_{n}\right\}$ can be obtained just making some assumption on the spectral behaviour of $T^{-1}$. Assume, for instance, that $T^{-1}$ is compact, then the sequence $\left\{\xi_{n}\right\}$ converges to 0 in the norm of $\mathcal{H}$, being the image of an orthonormal basis through a compact operator. Of course one can go further and require that $T^{-1}$ belongs to some other well-known classes of operators, giving a more accurate description of how fast $\left\|\xi_{n}\right\| \rightarrow 0$. For a discussion on this subject see [12].

\subsection{An Application}

The importance of Theorem 3.1 is that, once we have at hand a non self-adjoint operator $\mathrm{H}$, with purely discrete real spectrum, it is possible to construct the Hilbert space of the system by finding out exactly the closed operator defining an inner product which makes the eigenvectors of $\mathrm{H}$ orthonormal. As expected, the inner product of the Hilbert space can be given in terms of the metric operator $Q=T^{\dagger} T$ which is unbounded as an operator in $\mathcal{H}$, whereas is bounded as an operator in $\mathcal{H}_{+1}$ into $\mathcal{H}_{-1}$ (see Proposition 4.4 in Sect. 4). This change of domain is not a deal by the physical point of view, because the observable of the system are in general unbounded 
linear operators defined on a dense set $\mathcal{D}$ of Hilbert space $\mathcal{H}$. As an example of this situation, let us consider the Hilbert space $\mathcal{H}=L^{2}(\mathbb{R})$ and the Hamiltonian

$$
H=-\frac{d^{2}}{d x^{2}}+\frac{x^{2}}{2}-\frac{4 x}{1+x^{2}} \frac{d}{d x}-\frac{2}{1+x^{2}}=H_{0}+V
$$

where $H_{0}$ is the Hamiltonian operator of the harmonic oscillator and $V=-\frac{4 x}{1+x^{2}} \frac{d}{d x}-$ $\frac{2}{1+x^{2}}$ (in spite of the notation, the operator $V$ is not a physical potential, since it depends explicitly on the derivative operator). The set of its eigenvectors is $\left\{\xi_{n}=\frac{1}{\sqrt{2^{n} n ! \sqrt{\pi}}} H_{n}(x) \frac{e^{-\frac{x^{2}}{2}}}{1+x^{2}}, n \geq 0\right\}$, where $H_{n}(x)$ is the $n$th Hermite polynomial. The vectors $\xi_{n}$ 's do not form a orthonormal basis for $\mathcal{H}$. However, they constitute an $\omega$-independent complete Bessel sequence in $\mathcal{H}$ as we will see in a while. Hence, by Theorem 3.1, there exists a triplet of Hilbert spaces which has $\left\{\xi_{n}\right\}$ as a strict Riesz-like basis and, even more important, there exists a Hilbert space $\mathcal{H}_{+1}$ such that $\left\{\xi_{n}\right\}$ is an orthonormal basis for $\mathcal{H}_{+1}$ and such that $H \in \mathcal{B}\left(\mathcal{H}_{+1}\right)(H$ is closed and everywhere defined in $\mathcal{H}_{+1}$ ). Recall that, once we call $N_{n}=\frac{1}{\sqrt{2^{n} n ! \sqrt{\pi}}}$, the set $\left\{e_{n}(x)=N_{n} H_{n}(x) e^{-\frac{x^{2}}{2}}, n \geq 0\right\}$ is an orthonormal basis of $\mathcal{H}$. Hence the operator $T$ which takes the sequence $\left\{\xi_{n}\right\}$ into $\left\{e_{n}\right\}$ is $T=1+x^{2}$. This is an unbounded continuous operator defined on the dense set $D(T)=\left\{f \in \mathcal{H}:\left(1+x^{2}\right) f \in \mathcal{H}\right\}$, with bounded inverse: $T^{-1}=\frac{1}{1+x^{2}}$. The Hamiltonian $H$ is non self-adjoint and similar to $H_{0}$ by the intertwining operator $T, H=T^{-1} H_{0} T$, the eigenvectors of $H$ are transformed into those of $H_{0}$ and $H$ and $H_{0}$ have the same eigenvalues $\alpha_{n}=n+\frac{1}{2}$, for every $n \geq 0$ sorted $n$ by $n$; (in particular, the ground state $\xi_{0}$ is transformed in that one of $H_{0}$ ). It remains to show that $\left\{\xi_{n}\right\}$ is an $\omega$-independent complete Bessel sequence in $\mathcal{H}$. Indeed, $\left\{\xi_{n}\right\}$ is a Bessel sequence since there exists $C=\left\|T^{-1}\right\|>0$ such that for every finite sequence of complex numbers $\left\{c_{0}, c_{1}, \ldots c_{n}\right\}, n \in \mathbb{N}$,

$$
\left\|\sum_{k=0}^{n} c_{k} \xi_{k}\right\|^{2}=\left\|\sum_{k=0}^{n} c_{k} T^{-1} e_{k}\right\|^{2} \leq C \sum_{k=0}^{n}\left|c_{k}\right|^{2} .
$$

They are $\omega$-independent because if

$$
\sum_{n=0}^{\infty} c_{n} \xi_{n}=0=\sum_{n=0}^{\infty} c_{n} T^{-1} e_{n}=T^{-1}\left(\sum_{n=0}^{\infty} c_{n} e_{n}\right),
$$

then it implies $c_{n}=0$, for every $n \geq 0$, by the continuity and the injectivity of $T^{-1}$. Furthermore, they are a complete set because if $f \in \mathcal{H}$ is such that $\left\langle f \mid \xi_{n}\right\rangle=0$ for every $n$, then

$$
0=\left\langle f \mid \xi_{n}\right\rangle=\left\langle f \mid T^{-1} e_{n}\right\rangle=\left\langle T^{-1} f \mid e_{n}\right\rangle=0
$$


which by the injectivity of $T^{-1}$ implies $f=0$. Notice that, albeit $\left\{\xi_{n}=T^{-1} e_{n}\right\}$ is an $\omega$-independent complete Bessel sequence in $\mathcal{H}$, it is not a Riesz basis for $\mathcal{H}$ because $T=\left(1+x^{2}\right)$ is an unbounded operator. Now, following what we saw before, the natural space where considering the previous operator $H$ is $\mathcal{H}_{+1}=D(T)\left[\|\cdot\|_{+1}\right]$ with $\|\cdot\|_{+1}=\left\|\left(1+x^{2}\right) \cdot\right\|$.

\section{Operators Defined by Strict Riesz-Like Bases}

In this section some results in [13] are generalized to the case of operators defined in triplets of Hilbert spaces. Furthermore, we will prove some result about the similarity of operators introduced here, and a characterization of those which have real eigenvalues.

Let $\left\{\xi_{n}\right\}$ be a strict Riesz-like basis for the triplet $\mathcal{H}_{+1} \subset \mathcal{H} \subset \mathcal{H}_{-1}$ and $\left\{\zeta_{n}\right\}$ its dual basis. If $\boldsymbol{\alpha}=\left\{\alpha_{n}\right\}$ is a sequence of complex numbers we can formally define, for $f \in \mathcal{H}_{+1}$,

$$
\begin{aligned}
& A^{\alpha} f=\sum_{n=1}^{\infty} \alpha_{n}\left(\xi_{n} \otimes \bar{\zeta}_{n}\right) f=\sum_{n=1}^{\infty} \alpha_{n} \overline{\left\langle\zeta_{n} \mid f\right\rangle} \xi_{n} \\
& B^{\alpha} f=\sum_{n=1}^{\infty} \alpha_{n}\left(\zeta_{n} \otimes \bar{\xi}_{n}\right) f=\sum_{n=1}^{\infty} \alpha_{n}\left\langle f \mid \xi_{n}\right\rangle \zeta_{n} . \\
& R^{\alpha} f=\sum_{n=1}^{\infty} \alpha_{n}\left(\xi_{n} \otimes \bar{\xi}_{n}\right) f=\sum_{n=1}^{\infty} \alpha_{n}\left\langle f \mid \xi_{n}\right\rangle \xi_{n} \\
& Q^{\alpha} f=\sum_{n=1}^{\infty} \alpha_{n}\left(\zeta_{n} \otimes \bar{\zeta}_{n}\right) f=\sum_{n=1}^{\infty} \alpha_{n} \overline{\left\langle\zeta_{n} \mid f\right\rangle} \zeta_{n}
\end{aligned}
$$

Of course, these are the simplest operators that can be defined via $\left\{\xi_{n}\right\}$ and $\left\{\zeta_{n}\right\}$.

Remark 4.1 Before going further, a comment is in order. In [14] Balazs introduced the notion of Bessel multipliers (frame multipliers, Riesz multipliers) whose definition is apparently similar to those given above. To be more precise, if $\left\{\varphi_{n}\right\},\left\{\psi_{n}\right\}$ are Bessel sequences respectively in two Hilbert spaces $\mathcal{H}_{1}$ and $\mathcal{H}_{2}$, fix $\boldsymbol{m}=\left\{m_{n}\right\}$ a bounded sequence of complex numbers, the Bessel multiplier for the Bessel sequences above is an operator $M: \mathcal{H}_{2} \rightarrow \mathcal{H}_{1}$ defined by

$$
M=\sum_{n=1}^{\infty} m_{n}\left(\varphi_{n} \otimes \bar{\psi}_{n}\right) .
$$

The main differences with the operators in (10)-(11) is that the two sequences $\left\{\varphi_{n}\right\}$, $\left\{\psi_{n}\right\}$ are not necessarily biorthogonal (in particular, in [14, Corollary 7.5] a necessary 
and sufficient condition is given for $\left\{\varphi_{n}\right\},\left\{\psi_{n}\right\}$ to be biorthogonal), and moreover, as we shall see in a while, we will also deal with possibly unbounded sequences. Thus, the two notions are not directly comparable.

Let $\mathcal{H}_{+1} \subset \mathcal{H} \subset \mathcal{H}_{-1}$ be a triplet of Hilbert spaces and $\left\{\xi_{n}\right\}$ a strict Riesz-like basis for $\mathcal{H}_{+1}$.

Clearly, the operator formally defined by (10) can take values in $\mathcal{H}_{+1}$ or in $\mathcal{H}$ or even in $\mathcal{H}_{-1}$, following the different topologies that make the series on the right hand side convergent. It is clear that, if $f \in \mathcal{H}_{+1}$, then

$$
\sum_{n=1}^{\infty} \alpha_{n} \overline{\left\langle\zeta_{n} \mid f\right\rangle} \xi_{n} \text { converges in } \mathcal{H}_{-1} \Leftrightarrow \sum_{n=1}^{\infty}\left|\sum_{k=1}^{\infty} \alpha_{k} \overline{\left\langle\zeta_{k} \mid f\right\rangle}\left\langle\xi_{k} \mid \zeta_{n}\right\rangle_{-1}\right|^{2}<\infty
$$

Since $\left\langle\xi_{k} \mid \zeta_{n}\right\rangle_{-1}=\left\langle\xi_{k} \mid \xi_{n}\right\rangle$, for every $k, n \in \mathbb{N}$, we can conclude that

$$
A^{\alpha} f \in \mathcal{H}_{-1} \Leftrightarrow \sum_{n=1}^{\infty}\left|\sum_{k=1}^{\infty} \alpha_{k} \overline{\left\langle\zeta_{k} \mid f\right\rangle} G_{k, n}\right|^{2}<\infty
$$

where $\left(G_{k, n}\right)$ is the Gram matrix of the basis $\left\{\xi_{k}\right\}$; i.e., $G_{k, n}=\left\langle\xi_{k} \mid \xi_{n}\right\rangle$, for $k, n \in \mathbb{N}$. Differently from the standard case, the Gram matrix of $\left\{\xi_{k}\right\}$ need not be bounded.

Similarly, since $\left\{e_{n}\right\}$ is an orthonormal basis in $\mathcal{H}$, we have

$$
A^{\alpha} f \in \mathcal{H} \Leftrightarrow \sum_{n=1}^{\infty}\left|\sum_{k=1}^{\infty} \alpha_{k} \overline{\left\langle\zeta_{k} \mid f\right\rangle}\left\langle\xi_{k} \mid e_{n}\right\rangle\right|^{2}<\infty
$$

where, as before, $e_{k}=T \xi_{k}, k \in \mathbb{N}$.

Finally, as we shall see in Proposition 4.2,

$$
A^{\alpha} f \in \mathcal{H}_{+1} \Leftrightarrow \sum_{k=1}^{\infty}\left|\alpha_{k}\right|^{2}\left|\left\langle\zeta_{k} \mid f\right\rangle\right|^{2}<\infty
$$

Of course, analogous considerations can be made for the operators defined in (11), (12) and (13). It is worth remarking that for the operators $B^{\alpha}$ and $R^{\alpha}$ the series on the right hand side of (11), (12) may converge also for some $f \in \mathcal{H}_{-1}$.

Now we examine more closely one of the cases listed above. In particular, we will suppose $A^{\alpha} f \in \mathcal{H}_{+1}$, for every $f \in \mathcal{H}_{+1}$. Under this assumption, let us define

$$
\left\{\begin{array}{l}
D\left(A^{\alpha}\right)=\left\{f \in \mathcal{H}_{+1} ; \sum_{n=1}^{\infty} \alpha_{n} \overline{\left\langle\zeta_{n} \mid f\right\rangle} \xi_{n} \text { exists in } \mathcal{H}_{+1}\right\} \\
A^{\alpha} f=\sum_{n=1}^{\infty} \alpha_{n} \overline{\left\langle\zeta_{n} \mid f\right\rangle} \xi_{n}, f \in D\left(A^{\alpha}\right)
\end{array}\right.
$$




$$
\left\{\begin{array}{l}
D\left(B^{\alpha}\right)=\left\{\Psi \in \mathcal{H}_{-1} ; \sum_{n=1}^{\infty} \alpha_{n}\left\langle\Psi \mid \xi_{n}\right\rangle \zeta_{n} \text { exists in } \mathcal{H}_{-1}\right\} \\
B^{\alpha} \Psi=\sum_{n=1}^{\infty} \alpha_{n}\left\langle\Psi \mid \xi_{n}\right\rangle \zeta_{n}, \Psi \in D\left(B^{\alpha}\right)
\end{array} .\right.
$$

Then we have the following

$$
\begin{aligned}
& \mathcal{D}_{\xi}:=\operatorname{span}\left\{\xi_{n}\right\} \subset D\left(A^{\alpha}\right) ; \\
& \mathcal{D}_{\zeta}:=\operatorname{span}\left\{\zeta_{n}\right\} \subset D\left(B^{\alpha}\right) ; \\
& A^{\alpha} \xi_{k}=\alpha_{k} \xi_{k}, k=1,2, \ldots ; \\
& B^{\alpha} \zeta_{k}=\alpha_{k} \zeta_{k}, k=1,2, \ldots
\end{aligned}
$$

Hence, $A^{\alpha}$ and $B^{\alpha}$ are densely defined and have the same eigenvalues. As we will see, if $\alpha_{n} \in \mathbb{R}, \forall n \in \mathbb{N}$, they are one the adjoint of the other.

It worths noting that the operators $\left(T^{\dagger}\right)^{-1}$ and $\left(T^{-1}\right)^{\dagger}$ do coincide [15, Remark 3.2].

Before continuing, we recall that if $X: D(X) \subseteq \mathcal{H}_{+1} \rightarrow \mathcal{H}_{+1}$ is a closed map and $D(X)$ is dense in $\mathcal{H}_{+1}$, then there exists a closed densely defined map $X^{\dagger}: D\left(X^{\dagger}\right) \subseteq$ $\mathcal{H}_{-1} \rightarrow \mathcal{H}_{-1}$ such that

$$
\langle\Phi \mid X \xi\rangle=\left\langle X^{\dagger} \Phi \mid \xi\right\rangle, \quad \forall \xi \in \mathcal{H}_{+1}, \Phi \in \mathcal{H}_{-1}
$$

If $X$ is also closed as an operator in $\mathcal{H}$, then its Hilbert adjoint $X^{*}$ exists and $X^{*}=$ $X_{\mid D\left(X^{*}\right)}^{\dagger}$ where $D\left(X^{*}\right)=\left\{\phi \in \mathcal{H}: X^{\dagger} \phi \in \mathcal{H}\right\}$.

Proposition 4.2 The following statements hold.

(i) $D\left(A^{\alpha}\right)=\left\{f \in \mathcal{H}_{+1} ; \sum_{n=1}^{\infty}\left|\alpha_{n}\right|^{2}\left|\left\langle\zeta_{n} \mid f\right\rangle\right|^{2}<\infty\right\}$, $D\left(B^{\alpha}\right)=\left\{\Psi \in \mathcal{H}_{-1} ; \sum_{n=1}^{\infty}\left|\alpha_{n}\right|^{2}\left|\left\langle\Psi \mid \xi_{n}\right\rangle\right|^{2}<\infty\right\}$.

(ii) $A^{\alpha}$ and $B^{\alpha}$ are closed operators respectively in $\mathcal{H}_{+1}\left[\|\cdot\|_{+1}\right]$ and in $\mathcal{H}_{-1}[\| \cdot$ $\left.\|_{-1}\right]$.

(iii) $\left(A^{\alpha}\right)^{\dagger}=B^{\bar{\alpha}}$, where $\bar{\alpha}=\left\{\bar{\alpha}_{n}\right\}$.

(iv) $A^{\alpha}$ is bounded in $\mathcal{H}_{+1}$ if, and only if, $B^{\alpha}$ is bounded in $\mathcal{H}_{-1}$ and if, and only if, $\boldsymbol{\alpha}$ is a bounded sequence. In particular $A^{1}=I_{\mathcal{H}_{+1}}$ and $B^{1}=I_{\mathcal{H}_{-1}}$, where 1 is the sequence constantly equals to 1.

Proof (i): Since $\left\{\xi_{n}\right\}$ is an orthonormal basis for $\mathcal{H}_{+1}$, we have

$$
\left\|\sum_{k=n}^{m} \alpha_{k}\left\langle\zeta_{k} \mid f\right\rangle \xi_{k}\right\|_{+1}^{2}=\sum_{k=n}^{m}\left|\alpha_{k}\right|^{2}\left|\left\langle\zeta_{k} \mid f\right\rangle\right|^{2}, \quad f \in \mathcal{H}_{+1}
$$


which shows that $f \in D\left(A^{\alpha}\right)$ if and only if $\sum_{n=1}^{\infty}\left|\alpha_{n}\right|^{2}\left|\left\langle\zeta_{n} \mid f\right\rangle\right|^{2}<\infty$.

(ii): The proof of this statement can be made by slight modifications of [13, Proposition $2.1(2)]$.

(iii): It is easy to show that $B^{\bar{\alpha}}=\sum_{n=1}^{\infty} \bar{\alpha}_{n}\left(\zeta_{n} \otimes \overline{\xi_{n}}\right) \subseteq\left(A^{\alpha}\right)^{\dagger}$. Conversely, let $\Psi \in$ $D\left(\left(A^{\alpha}\right)^{\dagger}\right)$; then there exists $\Phi \in \mathcal{H}_{-1}$ such that

$$
\left\langle\Psi \mid \sum_{n=1}^{\infty} \alpha_{n} \overline{\left\langle\zeta_{n} \mid f\right\rangle} \xi_{n}\right\rangle=\langle\Phi \mid f\rangle, \quad \forall f \in D\left(A^{\alpha}\right)
$$

By (14) and (15), $\mathcal{D}_{\xi} \subseteq D\left(A^{\alpha}\right)$ and $A^{\alpha} \xi_{k}=\alpha_{k} \xi_{k}, k=1,2, \ldots$. Thus, $\left\langle\Psi \mid \alpha_{k} \xi_{k}\right\rangle=\left\langle\Phi \mid \xi_{k}\right\rangle, k=1,2, \ldots$. Hence

$$
\sum_{k=1}^{\infty}\left|\alpha_{k}\right|^{2}\left|\left\langle\Psi \mid \xi_{k}\right\rangle\right|^{2}=\sum_{k=1}^{\infty}\left|\left\langle\Phi \mid \xi_{k}\right\rangle\right|^{2}=\sum_{k=1}^{\infty}\left|\left\langle\left(T^{-1}\right)^{\dagger} \Phi \mid e_{k}\right\rangle\right|^{2}=\left\|\left(T^{-1}\right)^{\dagger} \Phi\right\|^{2}<\infty
$$

This implies that $\Psi \in D\left(B^{\bar{\alpha}}\right)$.

(iv): Let $\alpha$ be a bounded sequence, then there exists $M>0$ such that

$$
\left\|A^{\alpha} f\right\|_{+1}=\left\|\sum_{k=1}^{\infty} \alpha_{k} \overline{\left\langle\zeta_{k} \mid f\right\rangle} \xi_{k}\right\|_{+1} \leq M\left\|\sum_{k=1}^{\infty} \overline{\left\langle\zeta_{k} \mid f\right\rangle} \xi_{k}\right\|_{+1}
$$

hence $A^{\alpha}$ is bounded in $\mathcal{H}_{+1}$.

In a very similar way one can prove (i), (ii) and (iv) for $B^{\alpha}$. This completes the proof.

Remark 4.3 In [15] Di Bella, Trapani and the author gave a definition of spectrum for continuous operators acting in a rigged Hilbert space $\mathcal{D} \subset \mathcal{H} \subset \mathcal{D}^{\times}$. We refer to that paper for precise definitions and results. So a natural question is: what is the spectrum (in that sense) of the operator $A^{\alpha}$ defined above? Let us assume that the sequence $\boldsymbol{\alpha}$ is bounded, so that $A^{\alpha}$ is a bounded operator in $\mathcal{H}_{+1}$. The analysis is, in this case, particularly simple since, as usual, the set of eigenvalues consists exactly of the $\alpha_{k}$ 's and, if $\lambda$ does not belong to the closure $\overline{\left\{\alpha_{k} ; k \in \mathbb{N}\right\}}$ of the set of eigenvalues, then the inverse of $A^{\alpha}-\lambda I_{\mathcal{H}_{+1}}$ exists as a bounded operator in $\mathcal{H}_{+1}$. Hence, as expected, $\sigma\left(A^{\alpha}\right)=\overline{\left\{\alpha_{k} ; k \in \mathbb{N}\right\}}$. The situation for $B^{\alpha}$ is analogous.

Let us now consider the operators formally given by (12) and (13). They are, in fact, defined as follows:

$$
\left\{\begin{array}{l}
D\left(R^{\alpha}\right)=\left\{\Psi \in \mathcal{H}_{-1} ; \sum_{n=1}^{\infty} \alpha_{n}\left\langle\Psi \mid \xi_{n}\right\rangle \xi_{n} \text { exists in } \mathcal{H}_{+1}\right\} \\
R^{\alpha} \Psi=\sum_{n=1}^{\infty} \alpha_{n}\left\langle\Psi \mid \xi_{n}\right\rangle \xi_{n}, \Psi \in D\left(R^{\alpha}\right)
\end{array}\right.
$$




$$
\left\{\begin{array}{l}
D\left(Q^{\alpha}\right)=\left\{f \in \mathcal{H}_{+1} ; \sum_{n=1}^{\infty} \alpha_{n} \overline{\left\langle\zeta_{n} \mid f\right\rangle} \zeta_{n} \text { exists in } \mathcal{H}_{-1}\right\} \\
Q^{\alpha} f=\sum_{n=1}^{\infty} \alpha_{n} \overline{\left\langle\zeta_{n} \mid f\right\rangle} \zeta_{n}, f \in D\left(Q^{\alpha}\right)
\end{array}\right.
$$

It is clear that

$$
\begin{aligned}
& \mathcal{D}_{\zeta} \subset D\left(R^{\alpha}\right) \text { and } R^{\alpha} \zeta_{k}=\alpha_{k} \xi_{k}, k=1,2, \ldots ; \\
& \mathcal{D}_{\xi} \subset D\left(Q^{\alpha}\right) \text { and } Q^{\alpha} \xi_{k}=\alpha_{k} \zeta_{k}, k=1,2, \ldots
\end{aligned}
$$

Hence, $R^{\alpha}$ and $Q^{\alpha}$ are densely defined, and the following results can be established:

Proposition 4.4 The following statements hold.

(1) $D\left(R^{\alpha}\right)=\left\{\Psi \in \mathcal{H}_{-1} ; \sum_{n=1}^{\infty}\left|\alpha_{n}\right|^{2}\left|\left\langle\Psi \mid \xi_{n}\right\rangle\right|^{2}<\infty\right\}=D\left(B^{\alpha}\right)$, $D\left(Q^{\alpha}\right)=\left\{f \in \mathcal{H}_{+1} ; \sum_{n=1}^{\infty}\left|\alpha_{n}\right|^{2}\left|\left\langle\zeta_{n} \mid f\right\rangle\right|^{2}<\infty\right\}=D\left(A^{\alpha}\right)$.

(2) $R^{\alpha}$ and $Q^{\alpha}$ are closed.

(3) $\left(R^{\alpha}\right)^{\dagger}=R^{\bar{\alpha}}$ and $\left(Q^{\alpha}\right)^{\dagger}=Q^{\bar{\alpha}}$, where $\bar{\alpha}=\left\{\overline{\alpha_{n}}\right\}$.

(4) If $\left\{\alpha_{n}\right\} \subset \mathbb{R}$ (respectively, $\left\{\alpha_{n}\right\} \subset \mathbb{R}^{+}$) then $R^{\alpha}$ and $Q^{\alpha}$ are self-adjoint (respectively, positive self-adjoint). Furthermore, $R^{\alpha}$ is bounded from $\mathcal{H}_{-1}$ to $\mathcal{H}_{+1}$ if and only if $Q^{\alpha}$ is bounded from $\mathcal{H}_{+1}$ to $\mathcal{H}_{-1}$ and if, and only if, $\boldsymbol{\alpha}$ is a bounded sequence.

(5) If $\boldsymbol{\alpha}=\mathbf{1}$, where, as before, $\mathbf{1}$ denotes the sequence constantly equals to 1 , then $R:=R^{1}$ and $Q:=Q^{1}$ are bounded positive self-adjoint operators respectively of $\mathcal{B}\left(\mathcal{H}_{-1}, \mathcal{H}_{+1}\right)$ and of $\mathcal{B}\left(\mathcal{H}_{+1}, \mathcal{H}_{-1}\right)$ and they are inverses of each other, that is $R=(Q)^{-1}$, and $R=T^{-1}\left(T^{-1}\right)^{\dagger}, Q=T^{\dagger} T$, where $T \in \mathcal{B}\left(\mathcal{H}_{+1}, \mathcal{H}\right)$ is the operator such that $T \xi_{n}=e_{n}, \forall n \in \mathbb{N}$ and $\left\{e_{n}\right\}$ is an orthonormal basis for $\mathcal{H}$.

Proof The proof is similar to that of Proposition 4.2 and we omit it.

Remark 4.5 From Proposition 4.4, we see that there exists a bounded invertible, positive self-adjoint operator $Q$ from $\mathcal{H}_{+1}$ into $\mathcal{H}_{-1}$ that maps the strict Riesz-like basis $\left\{\xi_{n}\right\}$ into its dual basis $\left\{\zeta_{n}\right\}$.

Now, recall that $Q=Q^{1}$ and $R=R^{1}$, then we have the following

Proposition 4.6 Let $\boldsymbol{\alpha}=\left\{\alpha_{n}\right\}$ be a sequence of complex numbers. The following equalities hold:

$$
\begin{aligned}
& Q A^{\alpha}=B^{\alpha} Q=Q^{\alpha}, \\
& R B^{\alpha}=A^{\alpha} R=R^{\alpha} .
\end{aligned}
$$

Proof By Proposition 4.4 we have $D\left(A^{\alpha}\right)=D\left(Q^{\alpha}\right)$ and $D\left(B^{\alpha}\right)=D\left(R^{\alpha}\right)$. Moreover, from Proposition 4.2 and (18), if $f \in D(Q)$ 


$$
\begin{aligned}
Q f \in D\left(B^{\alpha}\right) & \Leftrightarrow \sum_{n=1}^{\infty}\left|\alpha_{n}\right|^{2}\left|\left\langle Q f \mid \xi_{n}\right\rangle\right|^{2}<\infty \\
& \Leftrightarrow \sum_{n=1}^{\infty}\left|\alpha_{n}\right|^{2}\left|\left\langle\zeta_{n} \mid f\right\rangle\right|^{2}<\infty \Leftrightarrow f \in D\left(Q^{\alpha}\right) .
\end{aligned}
$$

Similarly one proves the equality $D\left(Q A^{\alpha}\right)=D\left(Q^{\alpha}\right)$. It is easily seen that $Q A^{\alpha} f=$ $B^{\alpha} Q f=Q^{\alpha} f$, for every $f \in D\left(Q^{\alpha}\right)$. The proof of the second equality in (19) is analogous.

Remark 4.7 Equations (19) show that the two operators $A^{\alpha}$ and $B^{\alpha}$ are similar, in the sense that $Q$ and $R$ act as intertwining operators, see e.g. [5, Definition 7.3.1]. The intertwining relations between operators have found some recent interest in Quantum Mechanics.

A simple consequence of previous results is the following corollary which generalizes the Theorem by Mostafazadeh ${ }^{1}$ in [16] and thus gives a characterization of operators as $A^{\alpha}$ and $B^{\alpha}$ with real eigenvalues. Before continuing we recall the definition of (unbounded) quasi-Hermitian operator (see, e.g. [5, Definition 7.5.1]).

Definition 4.8 A closed operator $A$, with dense domain $D(A)$ is called quasiHermitian if there exists a metric operator $G$, with dense domain $D(G)$ in Hilbert space $\mathcal{H}$ such that $D(A) \subset D(G)$ and

$$
\langle A \xi \mid G \eta\rangle=\langle G \xi \mid A \eta\rangle, \quad \xi, \eta \in D(A)
$$

If $A$ is a quasi-Hermitian operator on $\mathcal{H}$, then by definition there exists an unbounded metric operator $G$ such that

$$
A^{\dagger} G=A G
$$

Corollary 4.9 Let $T$ be the operator which transforms the strict Riesz-like basis $\left\{\xi_{n}\right\}$ into an orthonormal basis of Hilbert space $\mathcal{H}$. The following statements are equivalent.

(i) The sequence $\boldsymbol{\alpha}=\left\{\alpha_{n}\right\}$ consists of real numbers.

(ii) $A^{\alpha}$ is quasi-Hermitian, with $G=Q=T^{\dagger} T$.

(iii) $B^{\alpha}$ is quasi-Hermitian, with $G=R=T^{-1}\left(T^{\dagger}\right)^{-1}$.

Proof $(i) \Rightarrow$ (ii) Suppose first that $\left\{\alpha_{n}\right\} \subset \mathbb{R}$, then according to (iii) of Proposition $4.2\left(A^{\alpha}\right)^{\dagger}=B^{\alpha}$. Then we can rewrite the first equality in (19) as

$$
Q A^{\alpha}=\left(A^{\alpha}\right)^{\dagger} Q
$$

hence $A^{\alpha}$ is quasi-Hermitian, with $G=Q$.

\footnotetext{
${ }^{1}$ The author in [16] calls the operators involved $G$-pseudo-Hermitian operators, however they are in fact quasi-Hermitian operators in the original sense of Dieudonné [17], even though unbounded.
} 
(ii) $\Rightarrow($ i $) \quad$ Let $A^{\alpha}$ be quasi-Hermitian, with $G=Q$ then

$$
A^{\alpha}=Q^{-1}\left(A^{\alpha}\right)^{\dagger} Q=T^{-1}\left(T^{\dagger}\right)^{-1} A^{\alpha} T^{\dagger} T .
$$

Put $H_{0}:=T A^{\alpha} T^{-1}$. It is an easy computation to prove that

$$
D\left(T A^{\alpha} T^{-1}\right)=D\left(\left(T^{\dagger}\right)^{-1}\left(A^{\alpha}\right)^{\dagger} T^{\dagger}\right)=\left\{f \in \mathcal{H} ; \sum_{n=1}^{\infty}\left|\alpha_{n}\right|^{2}\left|\left\langle f \mid e_{n}\right\rangle\right|^{2}<\infty\right\},
$$

and from (21) we have

$$
T A^{\alpha} T^{-1}=\left(T^{\dagger}\right)^{-1}\left(A^{\alpha}\right)^{\dagger} T^{\dagger}
$$

Since $D\left(\left(T^{\dagger}\right)^{-1}\left(A^{\alpha}\right)^{\dagger} T^{\dagger}\right) \subseteq D\left(\left(T A^{\alpha} T^{-1}\right)^{\dagger}\right)$ we can conclude that $H_{0}$ is symmetric and its eigenvalues are $\left\{\alpha_{n}\right\} \subset \mathbb{R}$.

$(i) \Leftrightarrow($ iii $) \quad$ is analogous to $(i) \Leftrightarrow(i i)$.

Other operators defined by a strict Riesz-like basis and its dual basis, more precisely lowering and raising operators, have been considered in [18] to factorize, under opportune hypotheses, the operators $A^{\alpha}$ and $B^{\alpha}$.

Acknowledgments The author is grateful to Prof. C.Trapani for his useful suggestions during the writing of this paper. This work was supported by the Gruppo Nazionale per l'Analisi Matematica, la Probabilità e le loro Applicazioni (GNAMPA) of the Istituto Nazionale di Alta Matematica (INdAM).

\section{References}

1. P.G. Casazza, The art of frame theory. Taiwanese J. Math. 4, 129-201 (2000)

2. O. Christensen, An Introduction to Frames and Riesz Bases (Birkhäuser, Basel, 2002)

3. C. Heil, A Basis Theory Primer (School of Mathematics, Georgia Institute of Technology, 1997)

4. G. Bellomonte, C. Trapani, Riesz-like bases in rigged Hilbert spaces. Zeitschrift für Analysis und ihre Anwendungen, to appear

5. J.P. Antoine, C. Trapani, Metric Operators, Generalized Hermiticity and Lattices of Hilbert Spaces, eds. by F. Bagarello, J.P. Gazeau, F.H. Szafraniek, M. Znoijl. Non-Selfadjoint Operators in Quantum Physics (Wiley, 2015), pp. 345-402

6. C.M. Bender, Making sense of non-Hermitian hamiltonians. Rep. Prog. Phys. 70, 947 (2007)

7. A. Mostafazadeh, Pseudo-Hermitian quantum mechanics with unbounded metric operators. Phil. Trans. R. Soc. A 371, 20120050 (2013)

8. A. Mostafazadeh, Pseudo-Hermitian representation of quantum mechanics. Int. J. Geom. Meth. Mod. Phys. 7, 1191-1306 (2010)

9. P. Siegl, D. Krejčiř́ik, On the metric operator for the imaginary cubic oscillator. Phys. Rev. D 86, 121702(R) (2012) 
10. H. Jarchow, Locally Convex Spaces (Teubner, Stuttgart, 1981)

11. R.H. Young, An Introduction to Nonharmonic Fourier Series (Academic Press, New York, 1980)

12. M.L. Arias, G. Corach, M. Pacheco, Characterization of Bessel sequences. Extracta Mat. 22, 55-66 (2007)

13. F. Bagarello, A. Inoue, C. Trapani, Non-self-adjoint Hamiltonians defined by Riesz bases. J. Math. Phys. 55, 033501 (2014)

14. P. Balazs, Basic definition and properties of bessel multipliers. J. Math. Anal. Appl. 325, 571-585 (2007)

15. G. Bellomonte, S. Di Bella, C. Trapani, Operators in rigged Hilbert spaces: some spectral properties. J. Math. Anal. Appl. 411, 931-946 (2014)

16. A. Mostafazadeh, Pseudo-Hermiticity versus PT-Symmetry II: a complete characterization of non-Hermitian hamiltonians with a real spectrum. J. Math. Phys. 43, 2814-2816 (2002)

17. J. Dieudonné, Quasi-Hermitian operators. Proceedings of the International Symposium on Linear Spaces, Jerusalem 1960 (Pergamon Press, Oxford, 1961), pp. 115-122

18. F. Bagarello, G. Bellomonte, On non-self-adjoint operators defined by Riesz bases in Hilbert and rigged Hilbert spaces. Proceedings of the 8th International Conference on Topological Algebras and their Applications (ICTAA-2014), to appear 